\title{
Oesophageal cancer in South Africa: The long timeline from onset of symptoms to definitive management
}

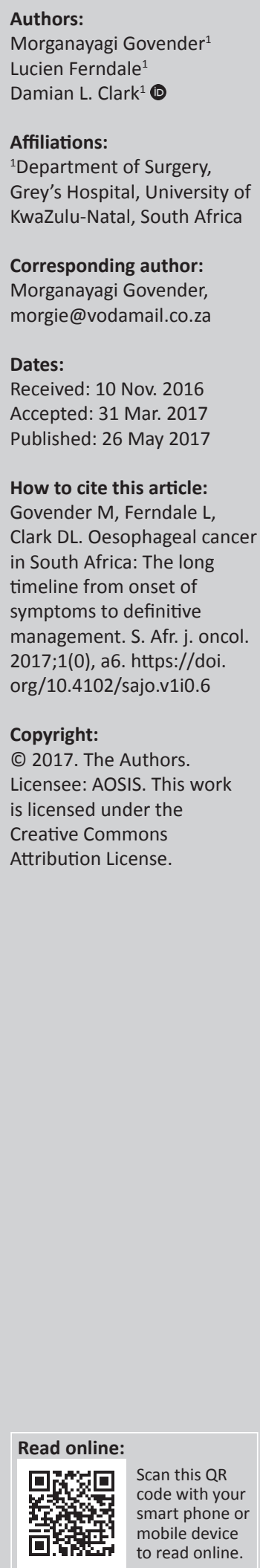

Background: In rural South Africa, most patients with oesophageal cancer have delayed presentations with debilitating symptoms and inoperable disease. This study was undertaken to quantify the delay between onset of symptoms and definitive treatment in a cohort of patients in rural South Africa, presenting to a state hospital in KwaZulu-Natal. The study also sought to establish reasons for delays in seeking medical attention and identify ways to encourage earlier presentation.

Methods: It was a two-armed study of patients with oesophageal cancer seen at Greys Hospital in Pietermaritzburg. One was a retrospective chart review establishing a timeline. The second part was a prospective study between June and November 2012 where data were collected by means of patient interviews.

Results: One hundred and thirteen charts were reviewed. The time from first symptoms to definitive management ranged from 2 to 14 months (average 7 months). Forty-six patients were interviewed. All experienced dysphagia but $83 \%$ were only prompted to seek help after weight loss. The duration of symptoms prior to first clinic or hospital attendance was 0-12 months (average 3 months). The reasons for the delay included the following: 41\% of patients did not consider dysphagia a significant symptom, $24 \%$ had no money, $19 \%$ sought the help of traditional healers first and $15 \%$ said the hospital was too far away.

Conclusion: There are long delays in the management of oesophageal cancer in our setting. The delays are prehospital as well as within the health care system. Lack of knowledge about oesophageal cancer symptoms and limited access to health care contributed to delays in management. Targeted quality improvement interventions are necessary. Patient education and improved referral systems are vital in encouraging earlier presentation.

\section{Introduction}

There is evidence to suggest that the referral system for acute surgical illness in South Africa is inefficient and this translates into poor outcomes for diseases such as acute appendicitis. ${ }^{1}$ There has not been much research done on the situation with regard to surgical oncology. Oesophageal cancer in South Africa is a significant problem. It is responsible for the second highest number of cancer-related deaths in the country and the most common cause of cancer-related mortality in African men. ${ }^{2}$ Despite advances in its diagnosis and management, the overall prognosis remains dismal. ${ }^{3,4,5,6}$ Most patients present late with inoperable disease and therapeutic interventions are directed at palliation rather than at cure. The objective of this study was to quantify the delay between the onset of symptoms and definitive treatment in our environment and to identify the reasons behind these delays. This study attempts to provide a timeline from the onset of symptoms of oesophageal cancer through to definitive care.

\section{Study design}

\section{Patients and methods}

It was a two-part study and both quantitative and qualitative methodologies were used. The first arm (A) was a retrospective chart review of all patients with oesophageal cancer diagnosed between January and November 2012. Patients were identified from combined records of the Oncology Clinic, the Surgical Outpatients Department (SOPD) and the Gastrointestinal Unit. The patient records were then retrieved, analysed and timeline plotted. This documented the time that elapsed between onset of symptoms, presentation at a health facility, time to diagnosis and time to definitive or palliative care. For the purpose of this study, diagnosis was made on endoscopic confirmation of tumour, and not on histology. The overall timeline from first symptom to definitive care was also plotted, and overall management for oesophageal cancer in these patients was also assessed viz. was there a standardised approach in the management. 
The second aspect of this study (B) was a detailed qualitative interview of a second cohort of patients with oesophageal cancer to assess their experience with the health system during the management of their tumour. This arm of the study was prompted by the preliminary findings of the chart review which revealed a delay between onset of symptoms and seeking help. Part B was designed to investigate this. It was undertaken between June and November 2012 and consisted of 46 consecutive patients seen with oesophageal cancer at the SOPD during this time period. Patient interviews were conducted in English or isiZulu depending on patient preference. A single interviewer and interpreter were used to eliminate bias. Each interview followed a standard proforma that sought detailed information on the onset of symptoms and the time to the first presentation to hospital. Specific questions were also asked to understand what prompted the patients to come to hospital and identify reasons for delays in presentation.

\section{Ethical considerations}

This study was conducted at Greys Hospital in Pietermaritzburg in 2012. Ethics approval was obtained from the University of KwaZulu-Natal Biomedical Research and Ethics Committee (UKZN BREC).

\section{Results \\ Chart review (Part A)}

A total of 113 charts were reviewed from January to December 2012. Of the 113 patients, $25 \%$ had been referred primarily to Oncology and $75 \%$ to Surgical Outpatients. A total of 3 underwent surgical resection of the primary, 6 underwent radiotherapy and 100 received a self-expanding metal stent for palliation. The average time from onset of symptoms to presentation was five months, from presentation to diagnosis was two months, from diagnosis to definitive management was one month. The average delay from onset of symptoms to provision of definitive care was seven months. This is summarised in Table 1.

Table 2 summarises the delays from presentation to the various investigations obtained and emphasises the

\begin{tabular}{lcc}
\multicolumn{1}{l}{ TABLE 1: Timeline of delay (derived from chart review). } \\
\hline Variable & Range & Average \\
\hline First symptoms & $0-12$ months & 5 months \\
Time to diagnose & $0-8$ months & 2 months \\
Definitive management & $0-7$ months & 1 month \\
Overall & $2-14$ months & 7 months \\
\hline
\end{tabular}

TABLE 2: Delay to individual investigations and non-standardised management (derived from chart review).

\begin{tabular}{lcc}
\hline Investigation & \% of patients & Average time to obtain \\
\hline Barium swallow & 18.5 & 2.0 months \\
Histology & 54.8 & 1.5 months \\
CT scan & 9.7 & 2.9 months \\
Oncology clinic review & 24.7 & 4.4 months \\
\hline
\end{tabular}

$\mathrm{CT}$, computerised tomography. non-standardised care. All patients underwent endoscopy, but only $54.8 \%$ had histological confirmation of malignancy. There was no clear algorithm followed and not all patients underwent imaging or referral to Oncology.

\section{Prospective qualitative assessment (Part B)}

Forty-six consecutive patients with oesophageal cancer who were treated at Greys Hospital, Pietermaritzburg, between June and November 2012 were interviewed by the primary author. The commonest symptoms were dysphagia (100\%), loss of weight $(91 \%)$, weakness $(50 \%)$ and abdominal pain $(13 \%)$. The symptoms that prompted presentation were weight loss (83\%) and dysphagia (17\%). This group experienced symptoms for an average of three months (range 1-12 months) before seeking attention. The following reasons were given for not seeking help earlier: lack of funds $(24 \%)$, need to seek traditional advice first $(19 \%)$ and distance to health care facility (15\%). Forty-one per cent did not consider dysphagia a significant enough symptom to prompt medical help.

\section{Discussion}

There is a growing body of work looking at inequalities in outcome of surgical diseases and there is evidence that economic status determines both health seeking behaviour and clinical outcome. ${ }^{1}$ This has been clearly demonstrated in acute surgical conditions in South Africa. ${ }^{1}$ The situation with surgical oncology is under-reported but there is evidence to suggest that rural patients with malignancy present late and have more advanced disease than other groups with similar disease processes. ${ }^{7,8,9}$ In this small cohort of South African patients, we have identified a that there is a significant delay from onset of symptoms to provision of definitive care.

There are two distinct areas in which these delays occurred. The areas are the pre-health system domain and the health system domain and it appears that both of these contribute to the overall delay. This is a significant finding because the strategies and avenues of research to address these deficits may be guided accordingly. In our series, it was found that a large proportion of patients did not seek medical contact until long after the onset of oesophageal symptoms. Seeking medical attention is influenced by a multitude of factors. These range from awareness of symptoms, varying educational levels, poor access to infrastructure, poverty, gender inequalities and cultural factors. ${ }^{9}$

Our data suggest that our cohort of rural patients tolerate dysphagia and unduly ignore its significance. Loss of weight was far more likely to precipitate health seeking behaviour than dysphagia, with only $17 \%$ presenting with dysphagia. This is a patient factor and should inform educational programmes aimed at raising awareness around oesophageal cancer. Another source of delay which has been documented in similar work from our institution on delays in acute appendicitis is the need to attend a traditional healer first. This once again raises a potential point of intervention and it 
may well be possible to develop targeted educational initiatives for traditional healers to teach them about socalled alarm symptoms. This simple strategy to redirect these patients into mainstream health care may prove invaluable. The socioeconomic factors of lack of funds and the long distances cited by our patients reflect structural inequalities in the society. As such, these barriers to health are difficult for clinicians to address.

Of concern are the delays within the health system that were revealed from our chart review. There appeared to be multiple areas of failure contributing to delays once the patient eventually did present to a health care provider (HCP). And these were either health system or human error related. Failure to refer to our institution timeously may have been either from inability to identify a red-flag symptom (human error on the part of the HCP) or failure to obtain an early appointment (system delay). Institutional bottlenecks once the patient presented to our hospital further compounded the situation. These system delays include long waiting times for barium studies, delays in obtaining histology, inappropriate referrals and failure to refer to Oncology. This haphazard management reflects the lack of formal algorithms for the investigation of oesophageal symptoms.

The long timeline is even more concerning when compared to similar data from other parts of the world. Table 3 compares our series with one each from India and Japan where time from symptoms to diagnosis and definitive management was also investigated. The difference is evident.

The unstructured management approach shown in our series for investigating oesophageal symptoms is problematic. In addition, the delays associated with each individual investigation contribute to the long delay to definitive treatment. It would seem that clinicians too require a raised awareness on alarm symptoms (dysphagia and weight loss) and the need to expedite referral and diagnosis. Outreach to peripheral hospitals and clinics where most patients make first contact may play a vital role in educating both patients and clinicians. A formal algorithm to standardise the approach to oesophageal symptoms is vital, but also needs to take resource limitations into consideration and not every patient may warrant a barium swallow or staging CT scan in our environment. A multidisciplinary team is vital to facilitate a standardised efficient approach to the management of oesophageal cancer. An enhanced investigative pathway needs to be developed in conjunction with radiology, oncology and surgery and this may well reduce the health care associated delays.

TABLE 3: Comparison to other parts of the world (in months).

\begin{tabular}{lccc}
\hline Variable & Our series & India $\mathbf{2 0 1 0}^{\mathbf{7}}$ & Japan $^{\mathbf{2 0 0 8}} \mathbf{8}^{\mathbf{2}}$ \\
\hline First presentation & 5 & - & 1.2 \\
To diagnosis & 2 & - & 1.4 \\
Definitive Mx & 1 & - & 0.25 \\
Overall & 7 & 3.7 & 2.1 \\
\hline
\end{tabular}

\section{Conclusion}

In this study, patients with oesophageal cancer experienced significant delays from the onset of symptoms to the time of definitive treatment. The factors contributing to these delays include barriers to health care and delays in assessment. There are also logistical problems with health care resources that need to be considered. It is our hope that highlighting and appreciating the significance of these factors can help develop targeted strategies to improve outcomes. Interventions need to be directed at both prehospital delays and delays within the health system. To this end, educational programmes around oesophageal alarm symptoms are necessary for the rural population, traditional healers and clinicians. Practical multi-disciplinary algorithms for the investigation and management of oesophageal symptoms need to be formalised as a matter of urgency. Only by fostering awareness around oesophageal cancer, encouraging earlier presentation and having clear management pathways can we hope to impact favourably on patient outcomes.

\section{Acknowledgements Competing interests}

The authors declare that they have no financial or personal relationships that may have inappropriately influenced them in writing this article.

\section{Authors' contributions}

M.G. was the primary author responsible for the concept of the study. This study formed part of her MMed Sc degree which was supervised by D.C. and M.G. performed the chart reviews, patient interviews and data analysis. L.F. assisted with literature review and proofreading. D.C. assisted with final editing of the article.

\section{References}

1. Kong V, Aldous C, Clarke DL. Understanding the reasons for delay to definitive surgical care of patients with acute appendicitis in rural South Africa. S Afr I Surg 2014;52(1):2-5.

2. Fitzmaurice C, Dicker D, Pain A, et al. The global burden of cancer 2013. JAMA Oncol. 2015;1(4):505-527. https://doi.org/10.1001/jamaoncol.2015.0735

3. Siegel R, Naishadham D, Jemal A. Cancer statistics, 2012. CA Cancer J Clin 2012;62:10-29. https://doi.org/10.3322/caac.20138

4. Dubecz A, Gall I, Solymosi N, et al. Temporal trends in long-term survival and cure rates in esophageal cancer: A SEER database analysis. J Thorac Oncol 2012;7:443447. https://doi.org/10.1097/JTO.0b013e3182397751

5. Berry MF. Esophageal cancer: Staging system and guidelines for staging and treatment. J Thorac Dis. 2014;6(Suppl 3):S289-S297.

6. Globocan 2012 [homepage on the Internet]. WHO International Agency for Research on Cancer. No date. [cited no date]. Available from: www.globocan. iarc.fr

7. Subasinghe N, Sindhia J. Delay in diagnosis of oesophageal cancer - Experience of a single unit from a developing country. Indian J Clin Oncol. 2010;47:151-155.

8. Wang J, Liu F, Gao H, et al. The symptom to treatment delay in cancer of oesophagus and stage at time of treatment. Japan J Clin Oncol. 2008;38(2):87-91. https://doi.org/10.1093/jjco/hym169

9. Ahmed SM, Adams AM, Chowdhury M, et al. Gender, socioeconomic development and health-seeking behaviour in Bangladesh. Soc Sci Med. 2000;51(3):361-371. https://doi.org/10.1016/S0277-9536(99)00461-X 